\title{
The effect of masker duration on forward and backward masking*
}

\author{
M. J. PENNER $†$ \\ Human Resources Laboratory, Williams Air Force Base, Chandler, Arizona 85224 \\ and \\ E. CUDAHY and G. W. JENKINS \\ Arizona State University, Tempe, Arizona 85281
}

\begin{abstract}
Temporal masking of clicks by noise was investigated using forward and backward masking paradigms. Both the noise duration and the temporal separation, $\Delta \mathrm{T}$, between the click and noise were varied. For very brief $\Delta$ Ts (100 microsec) and for very long $\Delta T s(100 \mathrm{msec})$, the duration of the masker did not greatly affect the click threshold. However, for intermediate $\Delta$ Ts $(3 \mathrm{msec})$, the threshold increased by as much as $44 \mathrm{~dB}$ as the noise duration increased from 0.1 to $100 \mathrm{msec}$. Temporal weighting functions, which describe the relative effectiveness of the noise as a function of $\Delta T$, were computed from these data.
\end{abstract}

A sinusoidal signal in noise is masked only by the nearby noise frequencies (Fletcher, 1940). The set of masking frequencies is called the critical band. Fletcher summarized this finding by postulating that the frequency analysis of the auditory system was accomplished by simple filter-like mechanisms. For sinusoidal signals in broadband noise, only the noise energy at frequencies passed by the filter is effective in masking the sinusoid. Many studies have attempted to specify the width and attenuation rate of these filters (Greenwood, 1961; for a review, see Patterson \& Green, 1973).

Recent research has indicated that filter-like mechanisms may also be fruitful in describing the temporal properties of the auditory mechanism. The argument is based on a temporal counterpart of the critical band. Investigators (Penner, Robinson, \& Green, 1972) found that a brief click is masked only by the noise in a limited region surrounding the click. That is, the noise occurring sufficiently before or after the click does not contribute to masking. By analogy with the critical band, the interval during which the noise is an effective masker was dubbed the critical masking interval. This finding may be summarized by postulating that the temporal analysis of the auditory system is accomplished by an averaging device or a temporal weighting function. For clicks in broadband noise, only the noise interval integrated by the function is effective in masking the click.

The attenuation rate, or skirts, of the temporal weighting function can be derived from the data gathered in forward and backward masking paradigms in

*This research was supported by a grant (No. 00000000 ) from the National institutes of Health and by the Human Resources Laboratory at Williams Air Force Base in Chandler, Arizona. The authors would like to thank Barry Leshowitz and Charles E. Robinson for their comments on earlier versions of the manuscript.

†Current address: Bell Telephone Laboratories, Holmdel, New Jersey 07733 . which the signal is a click and the masker is noise. As the click is moved closer to the noise, masking increases, presumably because the weighting function encompasses more noise. From the rate of increase of masking, the skirt of the weighting function can be determined. Thus, in this experiment, data from forward and backward masking paradigms are used to determine the skirts of the temporal weighting functions. Both the delay between the click and masker and the masker duration are varied.

\section{METHOD}

\section{Apparatus}

The signal, a click, was generated by a rectangular pulse lasting 100 microsec. The click was added to the white Gaussian noise, and the complex passed through a bandpass filter (Krohn-Hite 3550 ), whose low- and high-frequency cutoffs were 100 and $5,000 \mathrm{~Hz}$, respectively. The overall noise level was $88 \mathrm{~dB}$. The click spectrum was essentially flat up to $5,000 \mathrm{~Hz}$. The overall level of the click was measured by repeating it periodically and displaying it on an oscilloscope. Thus, a graphic estimate of the voltage of the click as a function of time was provided. These voltages were used to compute the click's energy.

\section{Procedure}

Masked thresholds were determined using a two-interval forced-choice procedure. Each trial began with a warning light. The first of the two observation intervals, spaced $1.0 \mathrm{sec}$ apart, occurred $0.5 \mathrm{sec}$ after the start of the trial. In each observation interval, a gated burst of noise was presented. In one of the two observation intervals, a click was presented with the noise. The interval containing the click was selected randomly. The listener indicated which observation interval he thought contained the click by pressing one of two buttons. The correct interval was indicated to the $O$ immediately after each response. Conditions were randomized daily.

Each session was diviced into eight 15 -min segments. Each segment, consisting of 140 trials, was devoted to determining a psychometric function for a given condition. An up-down psychophysical procedure was used for this purpose. The signal level varied from trial to trial over eight possible levels, spaced $3 \mathrm{~dB}$ apart. The signal, therefore, ranged over $21 \mathrm{~dB}$. Each threshold was determined from an 8-point psychometric 

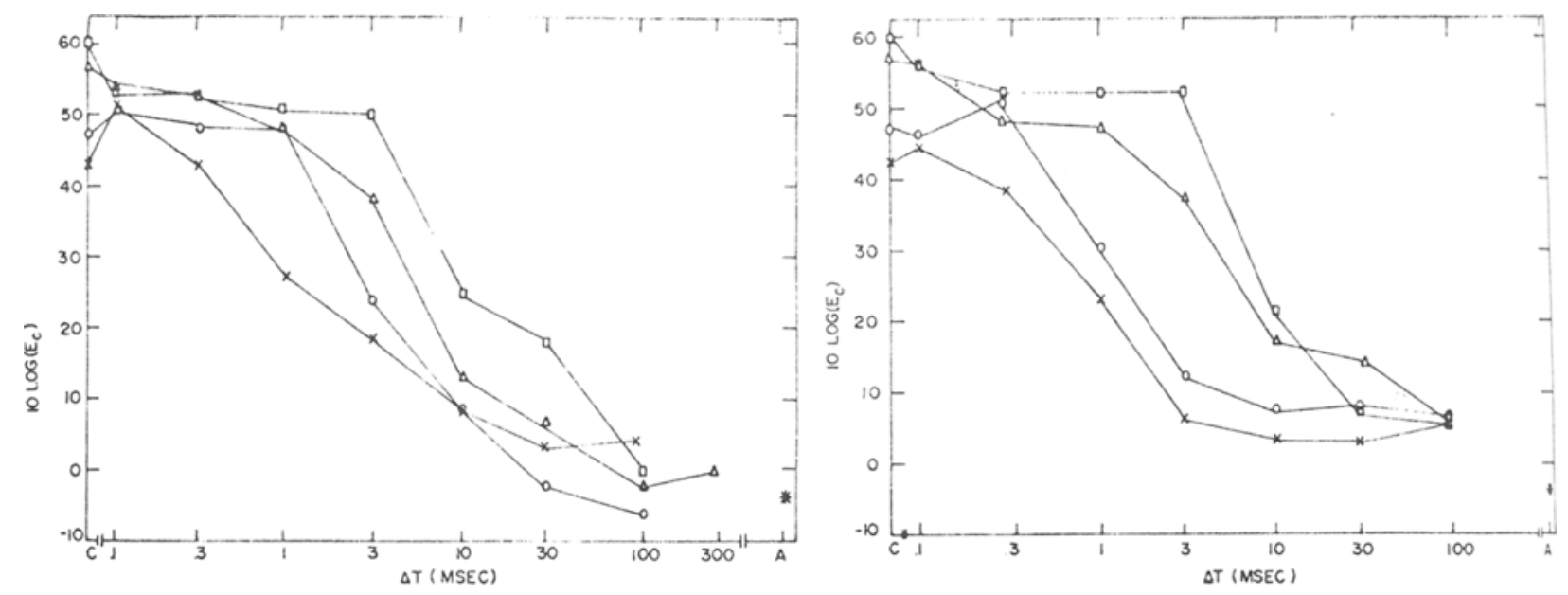

Fig. 1. The effect of masker duration on click threshold for various intervals between the click and noise. On the ordinate is the signal energy required for $75 \%$ correct detection. On the abscissa is the interval, $\Delta T$, between the onset or offset of the noise and the offset or onset of the click (in milliseconds). The Xs represent a $0.1-\mathrm{msec}$ noise, the circles a 1-msec noise, the triangles a 10-msec noise, and the squares a $100-\mathrm{msec}$ noise. The noise power per cycle per second, $\mathrm{N}_{\mathrm{o}}$, was $51 \mathrm{~dB}$ SPL. The $\mathrm{C}$ on the abscissa signifies the threshold of the click occurring in the center of a continuous noise. The $A$ on the abscissa signifies the absolute threshold of the click. The left-hand graph depicts forward masking conditions (masker followed by click), and the right-hand graph depicts the backward masking conditions (click followed by masker).

function. Except for the least and most attenuated signals, there were a minimum of 40 observations per point. At the extreme signal levels, there were a minimum of 20 observations per point.

Stimulus conditions were as follows. The noise duration was $0.1,1,10$, or $100 \mathrm{msec}$. The interval between the offset of the rectangular pulse and the onset of the noise (backward masking) was $0.1,0.3,1,3,10,30$, or $100 \mathrm{msec}$. The same intervals were employed in forward masking. Stimuli were presented diotically over TDH-39 headphones.

Two students attending Arizona State University served as listeners. The Ss were practiced Os who had served in a previous masking experiment. Since their thresholds were not significantly different, their data has been averaged.

\section{RESULTS}

The main novel finding is that the click threshold is affected by the duration of the noise masker. This finding is depicted in two figures. First, Fig. 1 presents the data from forward (on the left) and backward (on the right) masking conditions with the masker duration as the parameter. The abscissa is the temporal interval, $\Delta \mathrm{T}$, between the click and the noise. The ordinate is the level of the click required for $75 \%$ correct detection. In a backward masking condition with a $\Delta \mathrm{T}$ of $3 \mathrm{msec}$, it can be seen that, as the noise duration increases from $0.1 \mathrm{msec}$ (Xs in Fig. 1) to $100 \mathrm{msec}$ (squares), the click threshold increases by $44 \mathrm{~dB}$. On the other hand, for very brief $\Delta \mathrm{Ts}(0.1 \mathrm{msec})$ and for very long $\Delta \mathrm{Ts}$ $(100 \mathrm{msec})$, the click threshold is not greatly affected by the masker duration. This finding is also depicted in Fig. 2, in which the data in Fig. 1 are replotted with the noise duration on the abscissa. The parameter is the delay between the click and the noise. Again, forward masking conditions are displayed on the left and backward masking conditions on the right. For very brief delays (100 microsec) and very long delays
$(100 \mathrm{msec})$, the noise duration does not greatly affect the click threshold. For a 3-msec delay in the forward masking conditions, a 10 -fold increase in noise duration (from 1 to $10 \mathrm{msec}$ ) causes an increase of about $10 \mathrm{~dB}$ in click threshold. For a 3-msec delay in the backward masking conditions, a 10 -fold increase in noise duration (from 1 to $10 \mathrm{msec}$ ) causes an increase of about $20 \mathrm{~dB}$ in click threshold. Clearly, then, the increase in click threshold per 10-fold increase in noise duration depends on the delay. For delays examined in this experiment, the 3-msec delay resulted in the greatest increase (in click threshold per 10 -fold increase in masker durations) for both forward and backward masking.

Finally, the relative effect of forward and backward masking is shown in Fig. 3. In Fig. 3, the data from forward and backward masking conditions are compared. The duration of the masker was $0.1 \mathrm{msec}$. As can be seen, the click threshold is higher in forward than in backward masking, although the rate of decrease of masking appears to be about the same. Similar findings are obtained for the 1- and $10-\mathrm{msec}$ noise masker durations.

\section{DISCUSSION}

The effect of noise duration on click threshold depends on the interval between the click and the noise (see Figs. 1 and 2). For very brief (100-microsec) and for very long (100-msec) gaps between the click and the noise, the duration of the masking noise does not affect the click's threshold (see Fig. 2). For long gaps, the result is scarcely surprising, since, in essence, the task is similar to detection of a signal at absolute threshold. However, for the brief gaps, the results are extremely 

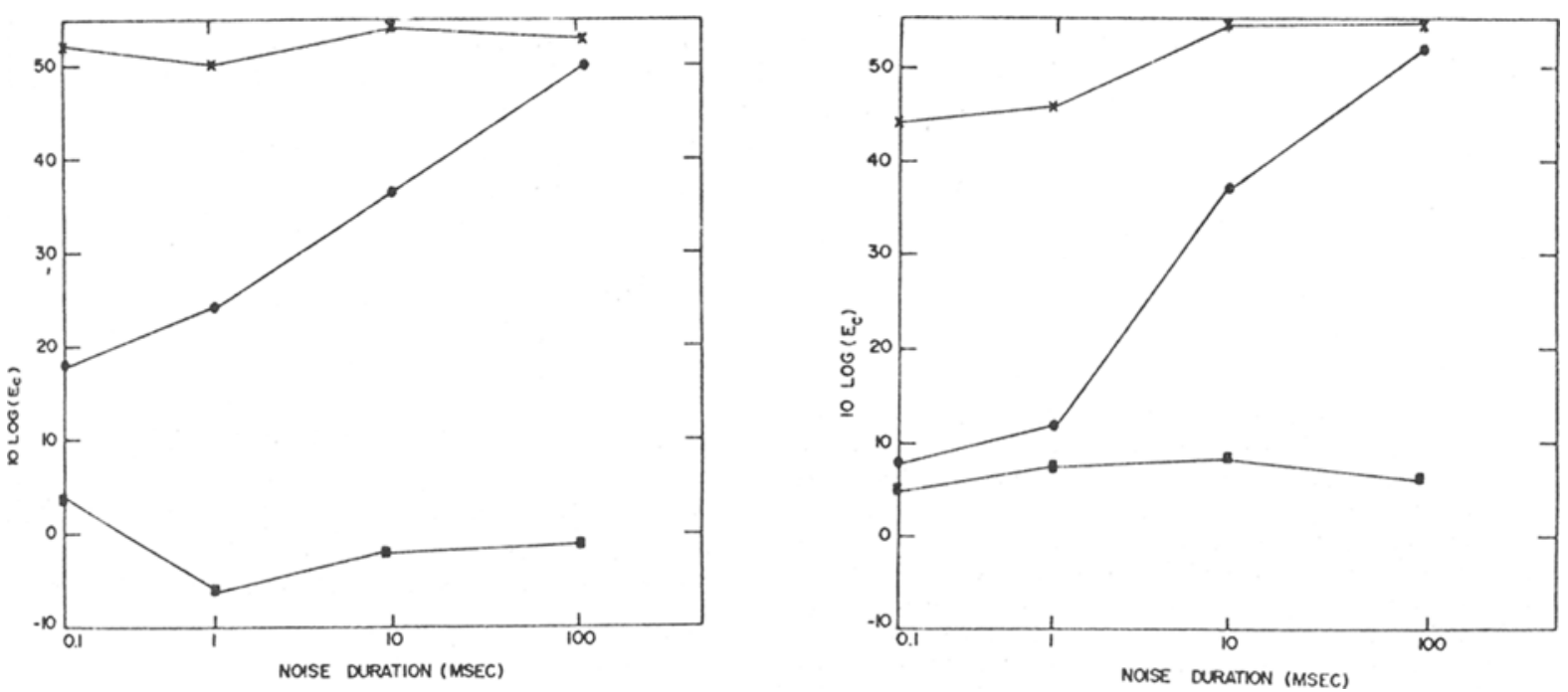

Fig. 2. The effect of masker duration on click threshold. On the ordinate is the signal energy required for $75 \%$ correct detection. On the abscissa is the noise duration (milliseconds). The parameter is the interval between the noise and the click. The Xs represent a 100 -microsec delay, the circles a 3 -msec delay, and the squares a $100-\mathrm{msec}$ delay. The left-hand graph depicts forward masking conditions, and the right-hand graph depicts backward masking conditions.

puzzling, since the task seems superficially similar to an increment detection experiment. If it were similar, then Weber's law might be expected to hold and an increase in masker duration would be accompanied by a concomitant increase in the click threshold. This does not appear to be the case. Zwicker and Fastil (1972) have recently presented identical findings for similar intervals between a tonal signal and a tonal masker in forward masking conditions, although Elliott (1964) has reported that the masker duration does not affect the signal threshold. Finally, for intermediate durations between the click and the noise, the click threshold increases as the noise duration increases. These findings lead to certain theoretical inferences concerning the skirts of the temporal weighting functions.

\section{Temporal Weighting Functions}

The data from the forward and backward masking conditions were used to derive the skirts of the temporal weighting function. There are two methods for calculating the skirts. One is appropriate for long noise masker durations and one is appropriate for very brief noise maskers.

\section{Temporal Weighting Functions for Long Masker Durations}

We now show that if the masker duration is much longer than the duration of the temporal weighting function, then the skirts of the temporal weighting function, $H(t)$, are determined by the derivative of the curve relating signal energy at threshold to the temporal interval between the click and the noise. For example, consider the $100-\mathrm{msec}$ noise masker. Since $100 \mathrm{msec}$ is about three times greater than previous estimates of the duration of the noise that masks the click (Penner et al, 1972), it seems reasonable to assume that $100 \mathrm{msec}$ is large compared to the duration of $H(t)$. If this is indeed the case, then the skirts of the temporal window can be simply determined, as is illustrated in Fig. 4.

Figure 4 depicts the temporal sequence of the signal and the preceding noise masker. A hypothetical temporal weighting function is also illustrated. The shaded area represents the overlap of the weighting

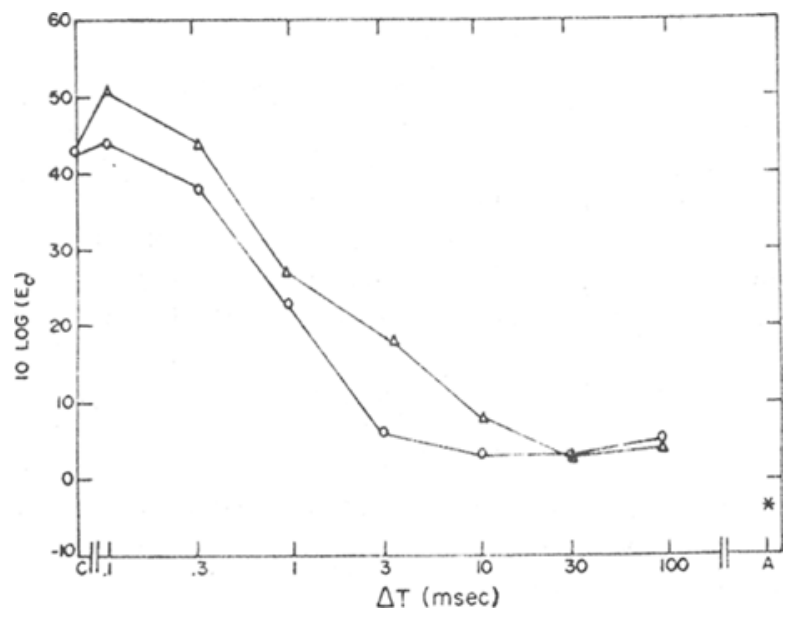

Fig. 3. A comparison of forward and backward masking. On the ordinate is the click energy required for $75 \%$ correct detection. On the abscissa is the interval between the offset of the click and the onset of the noise (backward masking, circles) or the offset of the noise and the onset of the click (forward masking, triangles). The data are from Fig. 1. The noise masker duration is $0.1 \mathrm{msec}$, and $N_{0}$ is $51 \mathrm{~dB}$. The $C$ on the abscissa signifies the threshold of the click occurring simultaneously with the masker. The $A$ on the abscissa signifies absolute threshold of the click. 


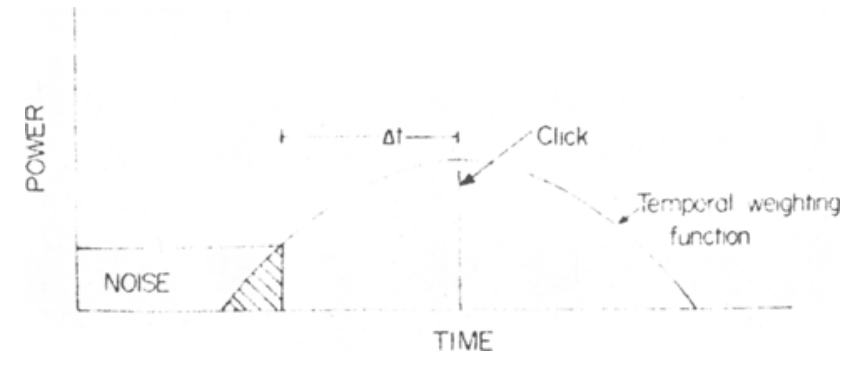

Fig. 4. This figure is a schematic diagram illustrating the temporal sequence of the click and noise maskers. On the ordinate is the average power. On the abscissa is time. Thus, the masker is noise with the same average power for some long time period, and the signal, the vertical line, is a click with power at essentially one point in time. The temporal weighting function of the hypothetical averaging device is also displayed. The shaded area represents the overlap of the weighting function and the noise. Thus, the shaded area represents the input noise which is effective in masking the click. As the gap, $\Delta t$, between the noise and the click decreases, the effective masking noise increases.

function and the noise masker. Thus, the shaded area represents the input noise which is effective in masking the click. As the gap between the noise and click decreases, the effective masking noise increases. By assumption, as long as the ratio of the energy of the click to the energy of the shaded area is large enough, the click is detected. If the energy of the click in a 1 -cycle band is $E_{o}$, the noise energy is $N_{o}(t)$, and the weighting function is $\mathrm{H}(\mathrm{t})$, then the model in Fig. 4 may be mathematically represented as

$$
E_{o}=k \int_{-\infty}^{\infty} N_{o}(t)|H(t)|^{2} d t .
$$

That is, the minimum energy, $\mathrm{E}_{\mathrm{o}}$, that a click must have to be heard in a noise, $N_{o}(t)$, is some constant proportion, $\mathrm{k}$, of the integral of the noise weighted by the square of the temporal weighting function. If the spectrum level of the noise is a constant $N_{o}$ for some long time, $t$, preceding the click and zero elsewhere (i.e., the noise occurs only until $t$ msec before the click) then

$$
E_{o}=k_{0} \int_{0}^{t}|H(t)|^{2} d t
$$

If the auditory filter is centered on the click and if $\mathrm{k}$ and $H(t)$ remain constant as $t$ is varied, then we may differentiate both sides of Eq. 2

$$
\frac{d}{d t} E_{o}=k N_{o} \frac{d}{d t} \int_{0}^{t}|H(t)|^{2} d t=k N_{o}|H(t)|^{2}
$$

Thus, the derivative of the curve relating click threshold to the temporal interval between the click and noise is proportional to the square of the temporal weighting function times a constant, $\mathrm{kN}_{\mathrm{o}}$.
Assuming that $100 \mathrm{msec}$ is sufficiently long, these arguments have been applied to the data in Fig. 1 for the $100-\mathrm{msec}$ noise masker. Smooth curves are fit to the data and the curves differentiated to obtain the following function,

$$
H(t)=\frac{1}{\left[\left(\frac{\Delta t}{\alpha}\right)^{2}+1\right]^{2}}
$$

for forward and backward masking $t \geqslant t_{o}$,

where $\Delta t$ is the interval between the click, occurring at time $t$, and noise, with offset at time $t_{o}$, and $\alpha=$ $1.3 \mathrm{msec}$. The $3-\mathrm{dB}$ down bandwidth is $1.29 \alpha$ and is $1.7 \mathrm{msec}$.

\section{Temporal Weighting Functions for Brief Masker Durations}

We now show that if the masker duration is much smaller than the duration of $\mathrm{H}(\mathrm{t})$, and if there is a constant signal-to-noise ratio at threshold, then the skirts of the temporal weighting function are given directly by the curve relating the click energy to the delay between the click and the noise. For example, consider the 100 -microsec noise masker. Since 100 microsec is about two orders of magnitude less than previous estimates of the duration of the noise that masks the click (Penner et al, 1972), it seems reasonable to assume that 100 microsec is negligible compared to the duration of $\mathrm{H}(\mathrm{t})$. If this is indeed the case, then the skirts of the temporal weighting function can be simply determined as illustrated in Fig. 5. Figure 5 shows the idealized power spectrum of the signal preceding the very brief noise masker. The temporal weighting function is also illustrated. Consider the effect on the click threshold as the noise moves from $t_{1}$ to $t_{2}$. By hypothesis, as the delay decreases from $t_{1}$ to $t_{2}, H(t)$ increases from its value at $t_{1}, H\left(t_{1}\right)$, to its value at $t_{2}, H\left(t_{2}\right)$. The ratio of the two values of the weighting function, $H\left(t_{2}\right) / H\left(t_{1}\right)$,

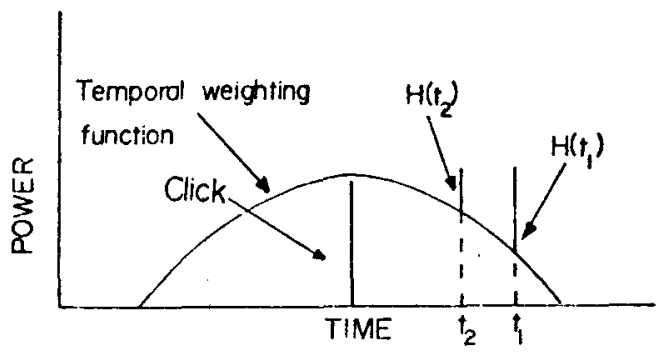

Fig. 5. This figure is a schematic diagram illustrating the temporal sequence of the click and the noise. On the ordinate is the average power. On the abscissa is time. The temporal weighting function of the hypothetical averaging device is also displayed. The weighting function is centered on the click. The dashed lines indicate the effective masker. It is assumed that as the masker moves from $t_{1}$ to $t_{2}$, the click threshold should increase by $10\left[\log H\left(t_{2}\right) / H\left(t_{1}\right)\right]$. 
determines the relative effect of the masker. In particular, if the ratio is $H\left(t_{2}\right) / H\left(t_{1}\right)$, then, by assumption, the click threshold must increase by $10\left[\log \mathrm{H}\left(\mathrm{t}_{2}\right) / \mathrm{H}\left(\mathrm{t}_{1}\right)\right]$ in order for detectability to remain constant. Hence, for very brief noise maskers, the change in click threshold as a function of the delay between the click and the noise is sufficient to determine the skirts of the temporal window.

Assuming that 100-microsec noise maskers are sufficiently brief, a smooth curve was fit to the data in Fig. 1 and the following function was obtained

$$
H(t)=\frac{1}{\left[\left(\frac{\Delta t}{\alpha}\right)^{2}+1\right]^{2}}
$$

for forward and backward masking $t \geqslant t_{o}$,

where $\Delta t$ is the interval between the click occurring at time and noise with offset of time $t_{o}$ in milliseconds and $\alpha=0.23 \mathrm{msec}$. The 3-dB down bandwidth is $1.29 \alpha$ and equals 296 microsec.

\section{Comment on the Calculated Weighting Functions}

The weighting functions (Eqs. 4 and 5) depend crucially on the duration of the noise masker: the half-power points for the 100 -msec masker are about 800 microsec, whereas the half-power points for the 100 -microsec click are about 150 microsec. A different weighting function is calculated for each noise duration. In particular, the weighting function appears to widen as the noise duration increases. This parallels an argument in the frequency domain: the critical bandwith, it is claimed, is originally wide, and narrows as the stimuli increase in duration. Likewise, it appears that the temporal weighting function is originally narrow, broadening as the stimuli increase in duration.

\section{CONCLUSION}

Studies of the critical masking interval led us to postulate that the temporal analysis of the auditory system might be accomplished by an averaging device whose shape could be inferred from the signal threshold in forward and backward masking conditions. The signal threshold in these conditions is known to depend on the noise level, $N_{o}$ (Elliott, 1962; Babkoff \& Sutton, 1968). We have found that it also depends on the noise duration. Since the signal threshold also depends on $\mathrm{N}_{\mathrm{o}}$, it seems reasonable to suppose that a different function would exist for each noise level as well. The functions might change considerably. In this study alone, the half-power points vary over nearly one-half an order of magnitude. It is apparently parsimonious, but untrue, to assume that there is a fixed temporal weighting function. Rather, there seems to be such adjustability that numerous weighting functions are conceivable.

Before abandoning the notion of a weighting function entirely, however, consider the results of calculations of the transfer functions for filters in the frequency domain. Here, too, it is claimed that the critical band, and hence the transfer function, depends on the stimulus's duration and amplitude. Despite the possibility of numerous functions, there is a convincing demonstration of the utility of a transfer function (Patterson \& Green, 1973). Patterson and Green's Ss are required to detect a sinusoid in the presence of low-pass and then high-pass noise. As the cutoff of the noise is moved closer to the sinusoid, the threshold of the signal rises. From the rate of increase, the filter's transfer function is computed. Patterson and Green then proceed to fit the data from other experiments (Egan \& Hake, 1950; Greenwood, 1961) with the derived filter. The success is remarkable. Whether their transfer function depends on the noise bandwidth, however, is unknown. Indeed, Patterson and Green's data give much narrower estimates of the critical band than do the two-tone data of Wegel and Lane (1924). Since an obvious difference in the two studies is the bandwidth of the masker, the noise bandwidth may well be a crucial factor. If the transfer function does depend on the noise bandwidth, then, as we find in the temporal domain, a whole family of transfer functions exists.

In conclusion, then, the notion of the invariance of the skirts of the temporal weighting function is not supported. Although it is mathematically possible to describe the skirts of the weighting functions, the plethora of functions indicates that the skirts of the temporal weighting function must be adjustable.

\section{REFERENCES}

Babkoff, H., \& Sutton, S. Monaural temporal masking of transients. Journal of the Acoustical Society of America, $1968,44,1373-1378$.

Egan, J. P., \& Hake, H. W. On the masking pattern of a simple auditory stimulus. Journal of the Acoustical Society of America, 1950, 22, 622-630.

Elliott, L. L. Backward masking: Monotic and dichotic conditions. Journal of the Acoustical Society of America, 1962, 34, 1108-1115.

Elliott, L. L. Backward masking: Different durations of the masking stimulus. Journal of the Acoustical Society of America, 1964, 36, 393(L).

Fletcher, H. Auditory patterns. Review of Modern Physics, 1940, 12, 47-61.

Green, D. M. Temporal auditory acuity. Psychological Review, 1971, 78, 540-551.

Greenwood, D. D. Auditory masking and the critical band. Journal of the Acoustical Society of America, 1961, 33, 484-502.

Patterson, R. D., \& Green, D. M. Auditory masking. In E. C. Carterette and M. P. Friedman (Eds.), Handbook of perception. New York: Academic Press, 1973, in press.

Penner, M. J., Robinson, C. E., \& Green, D. M. The critical 
masking interval. Journal of the Acoustical Society of America, 1972, 52, 1661-1668.

Penner, M. J., \& Cudahy, E. The critical masking interval: A temporal analog of the critical band. Journal of the Acoustical Society of America, 1973, 54, 1530-1534.

Ronken, D. Monaural detection of a phase difference between clicks. Journal of the Acoustical Society of America, 1970, 47, 1091-1099.

Wegel, R. L., \& Lane, C. E. The auditory masking of one pure tone by another and its probable relation to the dynamics of the inner ear. Psychological Review, 1924, 23, 266-285.

Wilson, R. H., \& Carhart, R. Forward and backward masking: Interactions and additivity. Journal of the Acoustical Society of America, 1970, 49, 1254-1263.

Zwicker, E., \& Fastil, L. H. On the dependence of forward masking on masker duration. Acustica, 1972, 26, 78-82.

(Received for publication June 11, 1973; revision received July $21,1973$. ) 